\title{
PRINCIPIOS Y PRECEPTOS DE LAS DOCTRINAS ECONÓMICAS
}

\author{
THE ECONOMIC DOCTRINES WITH THEIR PRINCIPLES AND HIS PRECEPTS \\ LUIS BORTESI LONGHI * \\ Docente Principal de la Facultad de Ciencias Contables
}

Universidad Nacional Mayor de San Marcos-UNMSM / Lima-Perú

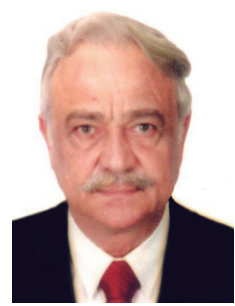

[Recepción: Setiembre de 2014/ Conformidad: Octubre 2014]

\section{RESUMEN}

Las doctrinas económicas constituyen los fundamentos éticos y técnicos que teóricamente resultan más convenientes, aconsejables y justos para acceder al desarrollo. Por lo tanto, la argumentación filosófica apunta a demostrar cuáles deben ser las prioridades que podrían impulsar y cuáles deben ser las estrategias de política económica para afianzar el progreso. Es así que, la estructura de una doctrina económica comprende tres capítulos:

$1^{\circ}$ Presupuestos o hipótesis de partida donde se enuncian las premisas básicas a priori. Este aspecto depende, naturalmente, de la formación intelectual del autor responsable de la doctrina.

$2^{\circ}$ Observaciones o comprobaciones fácticas del desenvolvimiento del modelo y sus efectos aplicativos.

$3^{\circ}$ Normas; es decir, la formulación de los preceptos cualitativos y filosóficos que inspiran la praxis y la ejecución institucionalizada del sistema económico.

\section{Palabras clave:}

Doctrina; sistema; desarrollo; normas; prioridades.

\begin{abstract}
The economic doctrines are the ethical and technical foundations that theoretically are more convenient, advisable and fair to access development. Therefore, the philosophical argument aims to demonstrate what should be the priorities that could drive and what should be the economic policy strategies to secure progress. So that the structure of an economic doctrine comprises three strands:

$1^{\circ}$ Budgets or starting hypothesis where basic a priori assumptions are set out. This aspect naturally depends, of the intellectual formation of the author responsible for doctrine.

$2^{\circ}$ Observations or findings of fact of the development of the model and its applications effects.

$3^{\circ}$ Standards; that is to say, the formulation of qualitative and philosophical precepts underlying the praxis and institutionalized execution of the economic system.
\end{abstract}

\section{Keywords:}

Doctrine; system; development; standards; priorities.

* Doctor en Ciencias Económicas. Email: luisbortesil@hotmail.com 


\section{INTRODUCCIÓN}

En el presente artículo se analizan los principales componentes de los fundamentos filosóficos de la economía, tales como: la dimensión humana del trabajo, la formación del capital, teoría de la utilidad, necesidades humanas, diversas formas de mercado, la institución del marketing, el instrumento de la técnica de la planificación económica, la intensidad de la intervención del Estado.

En el primer segmento del artículo, se realiza un breve recorrido de la historia de las doctrinas económicas, analizando las principales escuelas e intelectuales que cronológicamente se han sucedido y sus principales representantes: Mercantilismo, Fisiocracia, Escuela Clásica, Marxismo. Asimismo, se realiza un análisis particular sobre el pensamiento Keynesiano y también se consignan algunos representantes conspicuos del Neoclasicismo.

En el segundo segmento, se estudian los sistemas económicos más representativos vigentes y se examinan los aspectos favorables; así como, los posibles defectos de cada uno de ellos. Se trata de cuatro Sistemas Económicos paradigmáticos, a saber:

Sistemas Económicos Liberales o Capitalistas; Sistema Económico Socialista; Sistemas Económicos de Intervención o Economía Mixta y Economía Social de Mercado.

\section{BREVE RECUENTO CRONOLÓGICO DE ESCUELAS Y ECONOMISTAS NOTABLES}

\section{Mercantilismo}

Esta corriente de pensamiento que no estuvo muy integrada, tuvo lugar particularmente en los países de Europa Central durante los siglos XVI, XVII y la primera mitad del XVIII.

Sus principales fundamentos doctrinarios residen en dos premisas: la primera, postula que la principal tarea en materia económica debe ser la acumulación de metales preciosos, paralelamente, impulsando en forma concomitante el desarrollo del comercio exterior.

Se trata de un pensamiento que sostiene la necesidad de una intensa participación del Estado en todas las actividades económicas.
Un hecho curioso que podemos citar a propósito del mercantilismo, es que el conquistador Francisco Pizarro cuando desembarcó en la costa norte peruana, traía fijamente en su cabeza la importancia de los metales preciosos y prueba de ello, está el bochornoso episodio que se refiere al "cuarto del rescate" en Cajamarca. Se exigió una enorme cantidad de oro y plata para perdonarle la vida al inca Atahualpa. Sin embargo, fue vilmente traicionado.

Se puede aseverar que el pensamiento mercantilista acarreó hambruna en Europa y una prueba testimonial se encuentra en la famosa novela de la época, cuyo autor es anónimo, titulada "El Lazarillo de Tormes".

En resumen, consideramos que existe una relación analógica entre la leyenda mitológica de Midas y la corriente mercantilista. Midas, rey de Frigia, estableció una relación de culto con uno de los dioses menores del Olimpo, Dioniso y éste le concedió un deseo. El rey Midas le pidió que todo lo que tocase se convirtiera en oro y así, le fue otorgado el privilegio. Naturalmente, su arrepentimiento no tardó en llegar puesto que convertía en oro todo lo que tocaba, incluyendo a su propia hija y asimismo, alimentos y bebidas. Desfalleciente, invocó nuevamente a Dioniso para que le quitara la maldición. Compadecido el dios griego le indicó que se bañase en las aguas del río Pactolo el cual de acuerdo con la leyenda todavía arrastra pequeñas pepitas de oro.

\section{Fisiocracia}

A partir de la segunda mitad del siglo XVIII y en cierta manera, como reacción contestataria contra el mercantilismo, surge el pensamiento fisiocrático que sostiene que todo debe estar regido por el "orden natural". (Se tener en cuenta que "fisio" es un prefijo griego que significa "natural" como fisioterapia, fisiológico, etc.).

En este sentido, la escuela fisiocrática se opone rotundamente a la intervención del Estado y a su vez, postula como pensamiento central que la base de la riqueza de las naciones procede de las fuentes naturales; principalmente, de la agricultura.

Una síntesis notable de esta escuela de pensamiento doctrinario está en la famosa frase "Laissez 
Faire”, que fue acuñada por el economista francés Jean-Claude Marie Vincent de Gournay. La frase completa es: Laissez faire et laissez passer, le monde va de lui même; «Dejen hacer, dejen pasar, el mundo va solo».

La fisiocracia o fisiocratismo fue una escuela de pensamiento económico del siglo XVIII. Fundada por François Quesnay y Anne Robert Jacques Turgot en Francia. El origen del término fisiocracia proviene del griego y quiere decir "gobierno de la naturaleza", al considerar los fisiócratas que las leyes humanas debían estar en armonía con las leyes de la naturaleza. Esto está relacionado con la idea de que sólo en las actividades agrícolas, la naturaleza posibilita que el producto obtenido sea mayor que los insumos utilizados en la producción, surgiendo así un excedente económico. Los fisiócratas calificaron de estériles las actividades como la manufactura o el comercio, donde la incautación sería suficiente para reponer los insumos utilizados.

Uno de los aspectos más importantes de esta doctrina económica es que el Estado debe abstenerse de intervenir en las actividades productivas y comerciales, percibir una cuota mínima de impuestos y consagrarse, principalmente a lo que se llamó "El Estado Gendarme" donde se propone que la actividad estatal se circunscriba a mantener el orden.

\section{Los clásicos}

Dos pensadores importantes de la escuela clásica son Adam Smith y David Ricardo.

Adam Smith Douglas, célebre pensador escocés, profesor en la Universidad de Glasgow. En su cátedra original no era catedrático de economía sino de filosofía, y la asignatura a su cargo fue "Filosofía Moral". De hecho, su primera obra importante se titula "Teoría de los Sentimientos Morales".

Durante su trayectoria como profesor, fue contratado en 1763 por el poderoso aristócrata Charles Townshend quien ofreció a Smith una pensión vitalicia a cambio de que sirviera como tutor de su hijastro, el III Duque de Buccleuch, durante un viaje de tres años por Suiza y Francia. En este viaje conoció a los fisiócratas franceses (entre ellos, Quesnay y Turgot), que defendían la economía y política basada en la primacía de la ley natural, la riqueza y el orden, y se encontró con su viejo amigo David Hume. También, conoció a otros pensadores ilustrados como Voltaire, Benjamín Franklin, Diderot, D’Alembert y Necker.

Durante ese periplo, que resultó de una gran riqueza intelectual para Smith, escribió su famosa y fundamental obra: "An Inquire into the Nature and Causes of the Wealth of Nations" redactada en un inglés impecable y que en castellano, se traduce como "Una Indagación Acerca de la Naturaleza y las Causas de la Riqueza de las Naciones". En dicha publicación, Adam Smith en gran medida contestando los errores del mercantilismo, sostiene que la clave de la riqueza de las naciones radica en el trabajo y su correcta organización.

Es digno de destacarse un tema que por lo general se estudia de manera muy superficial y que, sin embargo, forma parte central del pensamiento Smithiano, cual es la teoría de la "mano invisible". Sostiene que al buscar su propio provecho individual cada persona, es conducida por una mano invisible que termina por satisfacer a la colectividad en su conjunto. Lo que sugiere en realidad Adam Smith en esta aseveración, es que es la providencia la que guía a los individuos.

David Ricardo fue un economista inglés de origen judío sefardí-portugués, miembro de la corriente de pensamiento clásico económico y uno de los más influyentes junto a Adam Smith. Continuó y profundizó el análisis del circuito de producción de la república, cuyo origen se remonta a Quesnay y al fisiocratismo. Es considerado uno de los pioneros de la macroeconomía moderna por su análisis de la relación entre beneficios y salarios, uno de los iniciadores del razonamiento que daría lugar a la ley de los rendimientos decrecientes y uno de los principales fundadores de la teoría cuantitativa del dinero. Es por ello, que es invocado por grupos de pensamiento económico muy diferentes, desde los neoclásicos a los marxistas ingleses.

\section{Karl Marx}

Existen dos dimensiones distintas, importantes y complementarias en el pensamiento Marxiano: Una es de carácter filosófico cuando analiza la lucha de clases y se alinea con el pensamiento de Hegel en el 
sentido de la tesis, antítesis y síntesis. En este sentido, Marx se refiere a la infraestructura que está compuesta por la clase trabajadora y la superestructura donde se ubican aquellos que mantienen el poder. Explica que la infraestructura avanza de manera mucho más dinámica que la superestructura y al querer mantener sus privilegios y adecuarse a los tiempos, sobreviene lo que se llama la lucha de clases. Hay que tener en cuenta que el perfil filosófico de Marx fue muy teórico y sobre todo que los pronósticos o profecías que advirtió, como que habría en un futuro una sociedad sin clases, no fue acertado.

La otra dimensión de la teoría expuesta por Marx en su obra fundamental "El Capital" es de carácter económico y se puede centralizar en el tema de la plusvalía. Hay que considerar que en el año 1765 con la introducción de la máquina de vapor que aplicó su descubridor James Watt se produjo una tragedia mundial denominada la Revolución Industrial que degradó la dimensión humana del trabajo para convertirlo en una mercadería; es decir, un bien sujeto a las leyes del mercado y a la oferta y la demanda. Con anterioridad el trabajo humano estaba hecho con dignidad pero con el advenimiento de la máquina es el ser humano el que se pone a su servicio.

Marx sostiene que el trabajo convertido a mercadería está sujeto a la ley de la oferta y la demanda, y que por lo tanto, tiene valor de uso y valor de cambio. En esa dirección de pensamiento sostiene que el capitalista, explotador, le otorga un mínimo valor de cambio al trabajador obteniendo él un inmenso valor de uso. De esa sustancial diferencia que él llama "plusvalía" se forma injustamente la acumulación de capital. Un colaborador notable y fiel de Carlos Marx fue el próspero empresario textil Friedrich Engels coautor del famoso manifiesto comunista.

\section{John M. Keynes}

John Maynard Keynes fue un economista británico, considerado como uno de los más influyentes del siglo XX, cuyas ideas tuvieron una fuerte repercusión en las teorías y políticas económicas.

La principal novedad de su pensamiento radica en considerar que el sistema capitalista no tiende al pleno empleo ni al equilibrio de los factores productivos, sino hacia un equilibrio que solo de forma accidental coincidirá con el pleno empleo. Keynes y sus seguidores de la posguerra destacaron no solo el carácter ascendente de la oferta agregada, en contraposición con la visión clásica, sino además, la inestabilidad de la demanda agregada, proveniente de los shocks ocurridos en mercados privados como consecuencia de los altibajos en la confianza de los inversores. La principal conclusión de su análisis es una apuesta por la intervención pública directa en materia de gasto público que permite cubrir la brecha o déficit de la demanda agregada. Está considerado también, como uno de los fundadores de la macroeconomía moderna.

Keynes fue un personaje muy polifacético, que además, de ser un economista teórico, cambió la consideración aplicada de la macroeconomía en el siglo XX.

Resulta digno de mención el hecho de que Keynes fuera el delegado del Reino Unido en la histórica reunión de Bretton Woods, en el estado de New Hampshire, poco antes de finalizar la Segunda Guerra Mundial donde se decidieron aspectos fundamentales del destino del planeta. El representante de Estados Unidos fue Harry Dexter White.

\section{Alfred Marshall}

Marshall fue el economista británico más brillante de su época. También, fue un profesor sobresaliente y ejerció una gran influencia sobre los economistas de aquella época. Su mayor contribución a la economía fue sistematizar las teorías económicas clásicas y el desarrollo del concepto de utilidad marginal. Subrayó la importancia del análisis minucioso y la necesidad de adecuar las teorías a los nuevos acontecimientos. Es considerado uno de los antecesores de la economía del bienestar. Entre sus obras destacan Principios de Economía (1890) e Industria y Comercio (1919).

El resultado de sus esfuerzos fue la denominada «síntesis neoclásica», base de la teoría económica. En 1890 publicó su obra capital, Principios de economía, que durante muchos años fue el principal libro de economía de todo el mundo. En el primer volumen de la obra compaginó conceptos de la economía clásica como riqueza, producción, trabajo, capital 
o valor con aportaciones de la escuela marginalista como utilidad y utilidad marginal. A los agentes de la producción (tierra, trabajo, capital) añadió un nuevo factor, el de la organización industrial.

En el segundo volumen realizó una exposición del funcionamiento de los mercados, un análisis de oferta y demanda y expuso su teoría del equilibrio parcial, de la formación de la oferta, la incidencia de los monopolios y la distribución de la riqueza nacional. Los problemas más destacados que analizó fueron el de la formación de los precios y la distribución de la renta.

(No hay que confundir a Alfred Marshall con el secretario de estado de Estados Unidos General George Catlett Marshall, impulsor de un vasto programa de reactivación, el famoso plan Marshall, para 16 países de Europa al cabo de la Segunda Guerra Mundial).

\section{Joseph A. Schumpeter}

Joseph Alois Schumpeter fue un destacado economista austro-estadounidense, ministro de Finanzas en Austria entre 1919 y 1920 y profesor de la Universidad de Harvard (Estados Unidos) desde 1932 hasta su muerte en 1950 . Se destacó por sus investigaciones sobre el ciclo económico y por sus teorías sobre la importancia vital del empresario, subrayando su papel en la innovación que determinan el aumento y la disminución de la prosperidad. Popularizó el concepto de destrucción creativa como forma de describir el proceso de transformación que acompaña a las innovaciones. Predijo la desintegración sociopolítica del capitalismo, que según él, se destruiría debido a su propio éxito.

\section{PRINCIPALES SISTEMAS ECONÓMICOS}

Antes de abordar el análisis de los cuatro sistemas económicos más significativos y vigentes, señalados en la introducción del presente documento, resulta aconsejable puntualizar y discutir cuáles son los puntos críticos más trascendentes que distinguen a un sistema de otro.

Un primer aspecto de singular importancia resulta en el hecho de juzgar cuál es el factor más importante de la producción. Si un político acredita que el factor más importante de la producción es el capital, naturalmente su perfil es liberal o "capitalista”. Mientras que si otro pensador considera que el factor más importante de la producción es el trabajo, aduciendo que el capital es trabajo acumulado, tiene una inspiración colectivista o "socialista".

Dicho esto, se puede agregar que las consideraciones más importantes que habrán de distinguir a un sistema económico de otro, son las siguientes:

- Definición del régimen de propiedad, que es el tema central.

- Formas y reglas de producción e inversión.

- Normas para la distribución de los excedentes.

- Marco financiero y tributario.

- Intensidad y medida de la intervención del Estado.

\section{Sistemas económicos capitalistas o liberales}

Las principales características de estos sistemas son las siguientes:

- Existe libertad: de iniciativa, empresarial, de asociación, de contratación.

- El capital es el principal factor de la producción (y no el trabajo).

- Los precios se fijan por caprichos de mercado.

- El Estado interviene como promotor y regulador, pero no como empresario.

- Las posibilidades de acumulación de fortuna son infinitas.

- El marketing desempeña una fuerza impresionante.

- El rasgo determinante es el individualismo y la competitividad.

Las críticas más importantes que acarrea este sistema serían las siguientes:

- Estímulo poderoso al enriquecimiento.

- Aspiración al lujo.

- La tecnología provoca serios problemas de empleo.

- El mercado y el marketing se vuelven perversos: existe la obsolescencia planificada.

- Se presentan severos casos de angustia (estrés).

- Se incentiva el consumismo.

- Los pobres corren gravemente el riesgo de desamparo 


\section{Sistemas económicos socialistas o colectivistas}

Las principales características son las siguientes:

- Los medios de producción de la riqueza son propiedad del Estado.

- El mercado y por lo tanto, los precios son íntegramente controlados por el Estado.

- No existe propiedad particular sobre la moneda extranjera.

- El comercio exterior está reservado al Estado.

- La primera prioridad es la satisfacción de las necesidades básicas de la población mayoritaria y la seguridad exterior.

Las ventajas y desventajas más notables con respecto a este modelo serían las siguientes:

- Gran desarrollo en el campo de la educación, la salud y el deporte.

- Estado fuerte: "Potencia".

- Consumo indispensable para los pobres asegurado.

- Élite de funcionarios públicos privilegiada.

- Sensación de opresión y control.

- Escasos incentivos en las aspiraciones de la vida material.

\section{Sistemas económicos nacionalistas}

Es preciso señalar que, este esquema fue el que siguió el Presidente Juan Velasco Alvarado durante el llamado "gobierno revolucionario de las fuerzas armadas", a partir del año 1968.

Sus principales características son las siguientes:

- También llamados de "economía mixta".

- El Estado controla las finanzas importantes.

- Existen restricciones en tenencia de moneda extranjera.

- Enormes e importantes empresas públicas.

- Se emprenden radicales reformas estructurales.

- El tamaño del Estado es muy grande.

Una evaluación crítica que pudiera merecer este sistema se podría sintetizar de la siguiente manera:

- Sistema proteccionista.

- Menor vulnerabilidad de la industria nacional.

- Proyectos de desarrollo formulados a largo plazo.
- Mayor conciencia de la "identidad nacional".

- Apoyo de la banca de fomento sectorial.

- Menor eficiencia y productividad.

- Menor libertad y opaca iniciativa privada.

- Restricciones y severos controles a la actividad privada.

- Escasez de productos y repuestos importados.

\section{Economía social de mercado}

Resulta oportuno señalar que este sistema fue impulsado en la Alemania de la posguerra y se le reputó como "El Milagro Alemán". Sus principales creadores fueron los socialdemócratas Ludwig Erhard y su canciller Konrad Adenauer.

A continuación, se esquematizan sus principales postulados:

- Es una doctrina intermedia en cuanto concierne al grado de participación del Estado.

- Este interviene directamente tan sólo como subsidiario.

- El mercado fija las reglas del precio, pero debe estar en armonía con el interés social.

- Una prioridad es acceder al pleno empleo.

- Se garantiza la justicia social en materia de salud, prevención, niñez, ancianidad, y sobre todo la calidad de la educación.

- Si bien la iniciativa privada es libre, el Estado controla los excesos y los defectos que afecten la estructura social.

La constitución política peruana vigente consagra a la economía social de mercado como su modelo oficial. En efecto, el artículo $58^{\circ}$ y $59^{\circ}$ prescriben lo siguiente:

"Art. 58 - La iniciativa privada es libre. Se ejerce en una economía social de mercado. Bajo este régimen, el Estado orienta el desarrollo del país y actúa principalmente, en las áreas de promoción de empleo, salud, educación, seguridad, servicios públicos e infraestructura".

"Art. 59- El Estado estimula la creación de riqueza y garantiza la libertad de trabajo y la libertad de empresa, comercio e industria. El ejercicio de estas libertades no debe ser lesivo a la moral, ni a la salud, ni a la seguridad pública. El Estado brinda oportunida- 
des de superación a los sectores que sufren cualquier desigualdad; en tal sentido, promueve las pequeñas empresas en todas sus modalidades".

\section{Comentario final}

Es muy importante subrayar que no existe en la práctica un único sistema económico óptimo, puesto que todos los sistemas presentan fortalezas y debilidades.

En consecuencia, el hecho de estar cambiando constantemente de dirección la política económica, adhiriendo primero un sistema y después a otro muy distinto, configura un grave error. Esa pendularidad resulta nefasta porque se avanza y se retrocede en un circuito vicioso donde el balance final es un cero rotundo.

En el Perú, hasta antes del año 1990, se experimentó constantemente esa contradicción, motivo por el cual se vivió en crisis tras crisis. En este sentido, una paradoja histórica notable está registrada cuando en el año 1968 el gobierno militar derroca al gobierno constitucional y emprende reformas radicales con el objeto de cambiar de raíz todas las estructuras.

Sin embargo, doce años después, el pueblo repone en las urnas, al Presidente que fue derrocado. Naturalmente, es el resultado de ir a "fojas cero".

\section{CONCLUSIONES}

1. Las doctrinas económicas constituyen el fundamento teórico y su aplicación práctica se formaliza en la política económica.

2. La política económica, a su vez, se diseña con el propósito de instituir un modelo o sistema económico.

3. No existe un sistema económico óptimo. Todos tienen ventajas y desventajas.
4. En la práctica no existe un sistema económico "puro", puesto que la frontera de cada uno de ellos, es una frontera permeable y porosa.

5. Lo importante radica en el hecho de escoger un modelo determinado, aprovechar sus ventajas y al mismo tiempo, gestionar en todo lo posible sus debilidades.

6. En el Perú, a partir de 1990 se está siguiendo una dirección, no única pero coherente y los resultados favorables están registrados en los índices y variables macroeconómicas.

\section{REFERENCIAS BIBLIOGRÁFICAS}

1. QUESNAY, François (1968) Tableau Économique. Bergman

2. TURGOT, Jacques (1991) Discursos Sobre el Progreso Humano. Tecnos

3. SMITH, Adam (1958) La Riqueza de las Naciones - México Fondo de Cultura Econ.

4. RICARDO, David (1959) Principios de Economía Política y Tributación. Fondo de Cultura

5. MARX, Karl (1959) El Capital. Crítica de la Economía Política. Fondo de Cultura

6. KEYNES, John M. (1943) Teoría General de la Ocupación el Interés y el Dinero - Fondo de Cultura Económica

7. (2004) The End of Laissez Faire - Great Minds Series

8. MARSHALL, Alfred (2006) Principios de Economía. Editorial Síntesis

9. (1957) Industria y Comercio. Editorial Aguilar

10. SCHUMPETER, Joseph A. (1956) Teoría del Desarrollo Económico - Editorial Claridad

11. (2002) Los Ciclos Económicos. Prensas Universitarias de Zaragoza 